\title{
Gender-associated factors for frailty and their impact on hospitalization and mortality among community-dwelling older adults: a cross-sectional population-based study
}

\author{
Qin Zhang ${ }^{1}$, Huanyu Guo ${ }^{1}$, Haifeng Gu ${ }^{1}$, Xiaohong Zhao ${ }^{\text {Corresp. } 1}$ \\ ${ }^{1}$ Department of Geriatrics, First Affiliated Hospital, School of Medicine, Zhejiang University, Hangzhou, Zhejiang, P.R. China \\ Corresponding Author: Xiaohong Zhao \\ Email address: xiaohongzhao88@yeahmca.cn
}

Background. Frailty associated with aging increases the risk of falls, disability, and death. We investigated gender-associated factors for frailty. Methods. Data of 3,079 geriatric subjects were retrieved from the National Health and Nutrition Examination Survey (NHANES) 2007-2010 database. After excluding 1,126 subjects with missing data on frailty, medical history and survival, data of 1,953 patients were analyzed. Main endpoints were frailty prevalence, mortality rates and causes of death. Results. Frailty prevalence was $5.4 \%$ in males, $8.8 \%$ in females. Significant risk factors for geriatric frailty in males were being widowed/divorced/separated, low daily total calorie intake, physical inactivity, sleeping > 9 hours, smoking and hospitalization history; and in females were obesity, physical inactivity, sleeping $<6$ hours, family history of diabetes and heart attack, and hospitalization history. Frail subjects had higher mortality rates ( $22.5 \%$ male; $8.5 \%$ female) than pre-frail ( $8.7 \%$ male; $6.4 \%$ female) and non-frail ( $5.4 \%$ male; $2.5 \%$ female). Main causes of death were heart diseases $(41 \%)$ and chronic lower respiratory diseases $(23.0 \%)$ in males and nephritis/nephrosis (32.3\%) and chronic lower respiratory diseases (17.6\%) in females. Discussion. Factors associated with frailty differ by gender, with higher frailty prevalence in females and higher mortality in males. Gender-associated factors for frailty identified in this study may be useful in evaluating frailty and guiding development of public health measures for prevention. Key Message: Common predictive factors for frailty among older adults of both genders, including more frequent previous hospitalizations, physical inactivity, and certain gender-associated factors for frailty, are consistent with results of other NHANES studies in which self-reported higher levels of illness and sedentary behavior were directly associated with frailty. 
1 Gender-associated factors for frailty and their impact on hospitalization and mortality

2 among community-dwelling older adults: a cross-sectional population-based study

3 Short running title: Gender-associated factors for frailty

4 Qin Zhang MD, Huan-yu Guo, MD, MS, Hai-feng Gu, MD, MS, Xiao-hong Zhao, MD, MS

5 Department of Geriatrics, First Affiliated Hospital, School of Medicine, Zhejiang University,

6 Hangzhou 310003, China

7 Correspondence to: Xiao-hong Zhao

8 Address: 79 Qingchun Road, Hangzhou, Zhejiang Province, 310003,China

9 Tel./fax: +8657187236808

10 E-mail: zqdorothy@163.com 
12 Background. Frailty associated with aging increases the risk of falls, disability, and death. We

13 investigated gender-associated factors for frailty.

14 Methods. Data of 3,079 geriatric subjects were retrieved from the National Health and Nutrition

15 Examination Survey (NHANES) 2007-2010 database. After excluding 1,126 subjects with

16 missing data on frailty, medical history and survival, data of 1,953 patients were analyzed. Main

17 endpoints were frailty prevalence, mortality rates and causes of death.

18 Results. Frailty prevalence was $5.4 \%$ in males, $8.8 \%$ in females. Significant risk factors for geriatric frailty in males were being widowed/divorced/separated, low daily total calorie intake, physical inactivity, sleeping $>9$ hours, smoking and hospitalization history; and in females were obesity, physical inactivity, sleeping $<6$ hours, family history of diabetes and heart attack, and hospitalization history. Frail subjects had higher mortality rates (22.5\% male; $8.5 \%$ female) than pre-frail (8.7\% male; $6.4 \%$ female) and non-frail (5.4\% male; $2.5 \%$ female). Main causes of death were heart diseases (41\%) and chronic lower respiratory diseases $(23.0 \%)$ in males and nephritis/nephrosis (32.3\%) and chronic lower respiratory diseases $(17.6 \%)$ in females.

Discussion. Factors associated with frailty differ by gender, with higher frailty prevalence in females and higher mortality in males. Gender-associated factors for frailty identified in this study may be useful in evaluating frailty and guiding development of public health measures for prevention.

Key Message: Common predictive factors for frailty among older adults of both genders, including more frequent previous hospitalizations, physical inactivity, and certain genderassociated factors for frailty, are consistent with results of other NHANES studies in which selfreported higher levels of illness and sedentary behavior were directly associated with frailty. 
INTRODUCTION

Frailty in older adults results from cumulative decline in physiological functioning and increased physical and mental vulnerability associated with aging, reducing the ability of older

37 adults to respond effectively to illness or trauma (Walston et al. 2006; Eeles et al. 2012; Clegg et

al. 2013). Frailty in older adults increases the risk for adverse outcomes associated with falls, delirium, and incontinence; complications of treatment with medications or procedures;

41 is associated with increased symptoms and complex diagnoses, their tolerance for medical interventions is diminishing simultaneously (Walston et al. 2006). However, while one-quarter to one-half of adults over age 85 are estimated to be frail and experience functional decline without

44 obvious stressors or illnesses, many older adults remain vigorous into their later years (Clegg et al. 2013).

Differences between frail and non-frail older adults have led to the development of

47 various screening tools to assess frailty risk and facilitate epidemiologic study. Some of these

48 screening tools are based on frailty models such as the phenotype model (Fried et al. 2001) and

49 cumulative deficit model (Mitnitski, Mogilner and Rockwood, 2001), or on functional

50 restrictions, although none of these reliably identify frailty associated with aging (Sternberg et al.

51 2011). A frailty index based on impairments of cognitive function, mood, motivation,

52 communication, mobility and incontinence, activities of daily living, nutrition, social resources,

53 and comorbidities, is highly predictive of institutionalization or death (Rockwood et al. 2006).

54 Various frailty indicator questionnaires have also been developed, including the Frail Elderly

55 Functional Questionnaire, which is sensitive to changes in status (Gloth et al. 1999).

56 Nevertheless, while the clinical utility of these screening tools remains limited, frailty must be 
57 diagnosed to help slow the progression to disability, institutionalization, and death (Miller et al. 58 2017).

Physical inactivity is one of the strongest risk factors for frailty along with aging (Ma et al. 2017). Body composition in men and fat percentage in women are also associated with

61 increased risk of frailty (Waters et al. 2012). The prevalence of frailty is found to be greater in

62 women (Waters et al. 2012; Ma et al. 2017). In a European study, the frailty-free years of women

63 were significantly fewer than those of men, influenced by both biological and socio-economic

64 factors (Romero-Ortuno, Fouweather and Jagger, 2014). Although women are noted to live

65 longer than men, their health status may be poorer due in part to environmental influences on

66 frailty and that women are affected more than men by lifestyle factors, increasing their

67 vulnerability to subcellular mechanisms that increase recovery time (Hubbard, 2015). Health and

68 mortality were affected negatively by smoking in both men and women, and smokers were frailer

69 than non-smokers; however, women who smoked lost their survival advantage (Wang et al.

70 2013). Differences in the frailty index for men (0.244) and women (0.278) may stem from

71 evolutionary design as well as biological and socio-behavioral factors such as, for example,

72 fitness frailty in men and fertility frailty in women (Hubbard, 2015).

We hypothesized that identifying gender-associated risks for frailty would be useful in

74 frailty assessment and may help to address biological and lifestyle factors that contribute to

75 frailty. Therefore, we aimed to investigate gender-associated risk factors for geriatric frailty and

76 their impact on hospitalization and mortality.

\section{PATIENTS AND METHODS}

\section{Data source}


79 The present study analyzed respondent data from the National Health and Nutrition Examination

80 Survey (NHANES), which was collected in two cycles (2007-2008 \& 2009-2010) by the Centers

81 for Disease Control and Prevention (CDC), National Center for Health Statistics (NCHS) in the

82 USA. All data were from the Public Data General Release File documents, CDC and NCHS, U.S.

83 Department of Health and Human Services, Hyattsville, MD, USA (Centers for Disease Control

84 and Prevention [CDC]. National Center for Health Statistics [NCHS], National Health and

85 Nutrition Examination Survey Data 2007-2008). The data are released for research purposes and

86 permission to use the data is granted to researchers by the NCHS.

\section{Ethical considerations}

88 Ethical approval of the NHANES program and signed informed consent by participants were obtained prior to data collection by NHANES, therefore, no further ethical approval and informed

90 consent were required for the present study. Additionally, all NHANES data released by the

91 NCHS are de-identified and the data remain anonymous during data analysis.

\section{Study population}

Data of 3079 community-dwelling geriatric subjects with mean age 73.8 years and $48.6 \%$ male from two cycles of NHANES data collection during 2007-2010 were eligible for inclusion. After excluding 1123 subjects with missing data on frailty (e.g., body weight, health status, fatigue,

96 difficulty walking a specific distance), disease history (e.g., asthma, diabetes, cancer, osteoarthritis, heart disease, stroke, emphysema, chronic bronchitis, renal failure, etc.), and 3 subjects without survival data, data of 1,953 subjects were analyzed. Differences between included and excluded subjects are shown in Supplementary Table 1A (Supplement A). The included subjects were significantly younger with greater percentages of non-Hispanic White, married/living with partner, normal BMI, higher education level, higher family 
103 also had less family/personal medical history of asthma, diabetes, heart attack, and less

104 hospitalization $<3$ times, mental health consultation, osteoporosis and steroid usage.

105 Main outcome measures

106 Primary endpoint: The primary endpoint of the present study was the prevalence of geriatric

107 frailty. All included subjects were categorized into non-frail (scored 0), pre-frail (scored 1-2) and

108 frail (scored 3-5) clusters according to the previously validated FRAIL Scale (Morley,

109 Malmstrom and Miller, 2012). Pre-frail is defined as individuals at risk for frailty who fulfill

110 some, but not all, frailty criteria. Five FRAIL scale parameters (fatigue, resistance, ambulation,

111 illness, and weight loss) are scored from 0 to 5, representing the gradually increased presence of

112 each parameter.

113 Secondary endpoints: The secondary endpoints were mortality rates of all subjects and the major

114 causes of death. NHANES respondents were linked to NDI mortality data through December

11531,2011 for this section of the survey report.

\section{Study variables}

117 Variables recorded for each case included patient demographics (age, gender, race/ethnicity,

118 marital status), family medical history (diabetes, asthma, heart attack/angina, osteoporosis);

119 health and medical conditions (self-reported osteoporosis, fracture, prednisone or cortisone use,

120 mental health consultation, hormone replacement therapy): socioeconomic status

121 (education level, family poverty income ratio, health insurance status); lifestyle or behavioral

122 factors (BMI, smoking history, alcohol use, milk consumption, vegetarian status, food allergy,

123 water devices use, physical activity, sleep duration); and dietary factors (total daily calorie

124 consumption, total daily macronutrients consumption, vitamin D insufficiency, iron deficiency). 
125 Male and female data were analyzed separately to obtain different unique risk factors for each 126 gender. Data were obtained as follows:

- Demographic data were collected in home visits by trained interviewers using the Family and Sample Person Demographics questionnaires and Computer-Assisted Personal Interviewing (CAPI) system (Confirmit Corp. New York, USA). Race/ethnicity was selfreported as Mexican American, Other Hispanic, Non-Hispanic White, Non-Hispanic Black, and Other Races, including multiracial.

- Family medical history data were self-reported using NHANES interviewer-administrated questionnaires (Medical conditions). and Sample Weights questionnaire. For the ratio of family income to poverty threshold, the range of values is continuous from 0-5; values of 5 and greater recorded as 5 .

- Lifestyle and behavior factors were collected separately as follows: Body Mass Index (BMI) data were based on "Body Measures" of NHANES Examination Protocol and were collected at the NHANES Mobile Examination Center (MEC) by trained healthcare technicians. BMI data were defined by World Health Organization (WHO) criteria as: underweight $\left(\mathrm{BMI}<18.5 \mathrm{Kg} / \mathrm{m}^{2}\right)$, normal $\left(\mathrm{BMI}=18.5 \sim 24.9 \mathrm{Kg} / \mathrm{m}^{2}\right)$, overweight $\left(\mathrm{BMI}=25 \sim 29.9 \mathrm{Kg} / \mathrm{m}^{2}\right)$, obese $(\mathrm{BMI} \geq 30.0$ $\mathrm{Kg} / \mathrm{m}^{2}$ ) (National Health and Nutrition Examination Survey (NHANES) Body Mass Index data collection. U.S. Department of Health and Human Services). Smoking History was recorded using interviewer-administered NHANES questionnaires (Smoking - Cigarettes Use). Respondents were categorized as current regular smoker or never regular smoker. Alcohol Use was evaluated according to amounts determined to increase health 

standard drinks per week on average or more than 3 drinks on any day for women; moderate drinkers consume 1 drink per day for women and up to 2 drinks per day for men. Occasional and non-drinkers are those who do not fall into the above criteria. scores (ratio of working metabolic rate relative to resting metabolic rate) (World Health Organization. Metabolic Equivalent of Task (MET) scores: http://www.who.int/chp/steps/GPAQ/en/ (Accessed February 12). One MET is defined as the energy cost of sitting quietly and is equivalent to a calorie consumption of $1 \mathrm{kcal} / \mathrm{kg} / \mathrm{hour}$. MET scores were calculated using intervieweradministered NHANES questionnaires (Physical Activity). The cutoff point for MET scores was established as $600 \mathrm{MET}-\mathrm{min} /$ week equal to a moderate intensity of physical activity based on WHO recommendation as described previously (Ainsworth et al. 2011). night on weekdays or workdays?" and recording the number of hours. The National Sleep Foundation (NSF) has updated recommendations for daily sleep amounts across the lifespan, including new ranges for each age group (National Sleep Foundation $[\mathrm{NSF}])$. Data were categorized as normal, short, and long sleep 
duration. For adults $>65$ years, sleeping 7 8 hours daily was considered appropriate and recommended. treatment devices use and vegetarian status.

178 - Dietary factors were collected by in-person interviews conducted in a private room at the NHANES Mobile Examination Center (MEC). Measuring guides (e.g., various glasses, bowls, mugs, drink boxes/bottles, household spoons, measuring cups/spoons, ruler, thickness sticks, bean bags and circles) helped participants report food amounts. Detailed dietary intake data included food consumed during previous 24 hours and total daily calorie \& macronutrients. Data were categorized based on Dietary Guidelines for Americans 2010 (U.S. Department of Agriculture and U.S. Department of Health and Human Services. Dietary Guidelines for Americans), including recommended intake and higher and lower intake by age and gender. Vitamin D insufficiency was evaluated based on serum 25-hydroxyvitamin D data from the NHANES Laboratory Data Protocol (National Health and Nutrition Examination Survey (NHANES) Laboratory Data Protocol) and respective interpretation of deficiency levels. For the present study, the cutoff point was established as $<40 \mathrm{nmol} / \mathrm{L}$, as previously described (Schleicher et al. 2016). Iron deficiency was evaluated based on Standard Biochemistry Profile from the NHANES Laboratory Data Protocol (National Health and Nutrition Examination Survey [NHANES] Laboratory Data Protocol). Normal ranges were 55-160 $\mu \mathrm{g} / \mathrm{dL}$ for men and 40-155 $\mu \mathrm{g} / \mathrm{dL}$ for women; deficiency was defined as lower than these ranges.

- Disease associations included medical conditions (osteoporosis, fracture, prednisone or cortisone use, female hormone replacement therapy, and mental health consultation) selfreported during interviewer-administered questionnaire data collection. 
- Hospitalization utilization was obtained using the Hospital Utilization and Access to Care questionnaire from the NHANES database administered in home visits by trained interviewers using the CAPI software interview system (Confirmit Corp., New York, months, categorized as no hospitalization, $\leq 3$ times and more than 3 times.

\section{Statistical analysis}

204

A multistage probability sampling design was used, employing a combined 4-year dietary dayone sample weight calculated according to the National Center for Health Statistics (NCHS) method and applied to all analyses. T-tests were used for continuous variables and chi-square tests were used for categorical variables. Weighted linear regression analysis was performed to test differences in continuous characteristics between participants clustered according to health status levels of non-frail, pre-frail, and frail, while logistic regression was performed for categorical parameters and was implemented to identify factors associated with health status levels. Significant variables revealed in univariate analysis were used to establish the final multivariable model. Differences and effectors of geriatric frailty were examined separately by gender. All analyses were performed based on the NHANES sampling design and appropriate weighting of participants in the statistical models. All statistical analyses were two-tailed at 0.05 significance level. All analyses were completed using SAS Version 9.3 (SAS Institute Inc., Cary, $\mathrm{NC}, \mathrm{USA})$.

\section{RESULTS}


219 In the present study, the prevalence of frailty was $5.4 \%$ in males and $8.8 \%$ in females. Prevalence 220 of pre-frailty ranged from $38.1 \%$ in males to $40.1 \%$ in females. In males, race, obesity, health 221 insurance, family histories, mental health consultation and prevalence of fracture were equally

222 distributed among the three clusters of non-frail, pre-frail and frail subjects. However, non-frail 223 subjects had younger age, greater percentage of married/living with partner, higher family 224 poverty/income ratio, higher education level, less presence of osteoporosis or steroid usage, and 225 more than three times less hospitalization history compared with pre-frail and frail subjects. 226 (Table 1). between the three clusters of males, which included participants with low daily total calorie intake, low daily total protein intake, low daily total fat intake, iron deficiency, physical inactivity, shorter sleep duration, and smoking status. (Table 2) similarities, however non-frail subjects had younger age, a greater percentage of normal BMI, more hormone replacement therapy, less family history of heart attack, less hospitalization history of over 3 times, less vitamin D insufficiency, less high C-reactive protein (CRP), more physical inactivity over 600 MET and shorter sleep duration compared with pre-frail and frail subjects. (Tables $1 \& 2$ ) study group (n=1953) according to "included" and "excluded" subjects. (Supplementary Table

239 1A; see Supplement A.)

\section{Causes of death}


241 Major causes of death in study subjects are shown in Table 3. A higher mortality rate (22.5\%) was

242 found in frail men compared with non-frail (5.4\%) and pre-frail (8.7\%) men. Among males, most

243 subjects in the non-frail and pre-frail clusters died of malignant neoplasms (43.2\% vs. 39.9\%).

244 Although the numbers are small $(n=11)$ in the frail cluster, the leading cause of death among frail

245 males was heart diseases (41\%). A higher mortality rate $(8.5 \%)$ was found in frail women

246 compared with non-frail (2.5\%) and pre-frail (6.4\%) women. Among females, most subjects in

247 the non-frail and pre-frail clusters died from other causes (56.6\% vs. $24.7 \%)$. Although the

248 numbers are small $(n=7)$ in the frail cluster, the leading causes of death among frail females were

249 nephritis, nephrotic syndrome and nephrosis (32.3\%). (Table 3)

250 Gender-related factors associated with frailty

Results of univariate analysis are summarized in Table 4. Age, marital status, family

poverty income ratio, education, health insurance coverage, daily macronutrient intake, high

CRP, iron deficiency, physical inactivity, inappropriate sleeping duration, smoking,

254 hospitalization history and history of osteoporosis were associated with frailty in males. After

255 controlling for the effects of other variables, multivariate analysis revealed that six factors were

256 still statistically significant in males, including widowed/divorced/separated $(\mathrm{OR}=1.417,95 \%$

257 confidence interval [CI]: $1.041-1.929, \mathrm{p}=0.027)$, daily total calorie intake $(\mathrm{OR}=1.678,95 \% \mathrm{CI}$ :

$2581.072-2.625, \mathrm{p}=0.023)$, physical inactivity $(\mathrm{OR}=2.011,95 \% \mathrm{CI}: 1.424-2.838, \mathrm{p}<0.001)$, sleeping

259 hours exceeding 9 hours $(\mathrm{OR}=1.568,95 \% \mathrm{CI}: 1.005-2.447, \mathrm{p}=0.047)$, smoking $(\mathrm{OR}=2.219,95 \%$

260 CI: 1.616-3.046, $\mathrm{p}<0.001)$ and hospitalization history $(\mathrm{OR}=2.539,95 \% \mathrm{CI}: 1.721-3.745, \mathrm{p}<$

261 0.001). (Figure 1A)

In female subjects, univariate analysis revealed that age, obesity, family poverty/ income

ratio, education, daily macronutrient intake, vitamin D insufficiency, high CRP, moderate alcohol use, physical inactivity, shorter sleep duration, hormone replacement therapy, family history of 
265 diabetes and heart attack, and hospitalization history were associated with frailty. After

266 controlling for the effects of other variables, multivariate analysis revealed that only eight factors

267 were still statistically significant, including obesity $(\mathrm{OR}=1.685,95 \% \mathrm{CI}: 1.085-2.678, \mathrm{p}=0.020)$,

268 high CRP $(\mathrm{OR}=2.735,95 \% \mathrm{CI}: 1.660-4.504, \mathrm{p}<0.001)$, physical inactivity $(\mathrm{OR}=1.974,95 \% \mathrm{CI}$ :

$2691.297-3.004, p=0.002)$, sleep duration less than 6 hours $(\mathrm{OR}=1.535,95 \% \mathrm{CI}: 1.078-2.187, \mathrm{p}=$

$2700.018)$, family history of diabetes $(\mathrm{OR}=1.345,95 \% \mathrm{CI}: 1.024-1.767, \mathrm{p}=0.033)$, family history of

271 heart attack $(\mathrm{OR}=2.071,95 \% \mathrm{CI}: 1.535-2.794, \mathrm{p}<0.001)$, and history of hospitalization $(\mathrm{OR}=$

$2723.182,95 \%$ CI: 2.165-4.678, $\mathrm{p}<0.001)$. In contrast, higher family poverty/income ratio was

273 associated with lower risk of frailty $(\mathrm{OR}=0.828,95 \% \mathrm{CI}: 0.732-0.936, \mathrm{p}=0.003)$. (Figure $1 \mathrm{~B})$

\section{DISCUSSION}

\section{Main findings of this study}

276 In the present study, the prevalence of frailty was higher in females than in males and increased

277 with age in both sexes. Higher mortality rates were also found in men and women categorized as

278 frail, and mortality was higher in men. Although the numbers are small $(n=11)$ in frail men, the

279 leading cause of death was heart diseases, followed by chronic lower respiratory diseases; non-

280 frail and pre-frail men died of malignant neoplasms. The numbers were also small $(\mathrm{n}=7)$ in frail

281 women, and the leading causes of death were nephritis, nephrotic syndrome and nephrosis,

282 followed by chronic lower respiratory diseases, while non-frail and pre-frail women died from

283 other causes. Independent factors associated with frailty differed between genders. Men who

284 were widowed/divorced/separated, had lower daily total calorie intake, were physically inactive,

285 slept more than 9 hours, smoked and were hospitalized previously were more likely to be frail.

286 Frailty was more likely in women who were obese, had elevated CRP indicating inflammation, 
287 were physically inactive, slept less than 6 hours, were hospitalized previously and had a family

288 history of diabetes or heart attack. Higher family income/poverty ratio was associated with lower 289 risk of frailty in both genders.

290 What is already known on this topic

291 Frailty is understood as an increased risk of adverse health outcomes associated with aging. The 292 causes of frailty are multifactorial, including different types of compromised function and repair 293 processes (Miller et al. 2017). The physiological framework that explains frailty involves 294 vulnerabilities associated with aging and chronic disorders; that is, disability in older adults stems 295 mainly from the aging process itself, including unhealthy lifestyles and health disorders and a 296 reduced ability to respond to life's stressors (Rodriguez-Manas and Fried, 2015). Measuring 297 frailty is most often done using the frailty phenotype model and the frailty index. The FRAIL 298 scale (Morley, Malmstrom and Miller, et al. 2012) used in the present study incorporates aspects 299 of both but discriminates frailty at the lower prevalence levels (Blodgett et al. 2015b). Using the 300 5-item self-reported questionnaire (FRAIL scale) (Morley, Malmstrom and Miller,. 2012), has 301 been shown to estimate frailty prevalence accurately in community-dwelling older adults 302 (Yamada and Arai 2015). Consistent with our results, the prevalence of frailty is higher among 303 women and increases with age in both genders (Rodriguez-Manas and Fried, 2015). Development 304 of frailty in women is influenced by deficits of various hormones during aging and increased 305 inflammatory states (Eichholzer et al. 2013). Overlap in frailty, disability, and comorbidities is a 306 combination of causes contributing to mortality, and overlap is associated especially with greater 307 frailty (Theou et al. 2012). Prevention is possible. A research team conducting a 10-year 308 community intervention for frailty prevention among older adults $(>65)$ used a public health 309 approach with community consensus to develop a health education program that effectively 
310 promoted healthy aging; the success of the program relied on comprehensive assessment of

311 geriatric individuals and improving self-care ability (Shinkai et al. 2016).

\section{What this study adds}

313 We found common predictive factors for frailty among older adults of both genders, including 314 more frequent previous hospitalizations and physical inactivity, consistent with results of other

315 NHANES studies in which self-reported higher levels of illness and sedentary behavior were 316 directly associated with frailty (Blodgett et al. 2015a). Notably, non-frail older adults of both 317 genders had a higher family income/poverty ratio, which gradually decreased toward the frail 318 group, indicating that greater financial security helps people take better care of their health.

319 However, independent risk factors for frailty showed that, for men, being widowed, divorced, or 320 separated increased the likelihood of frailty along with low daily total calorie intake, sleeping too 321 much, and smoking, suggesting that men living alone take poor care of their nutritional needs and 322 persist in harmful lifestyle habits. Correspondingly, better diet quality is associated with lower 323 odds of developing frailty (Chan, Leung and Woo, 2015). In women, obesity, inflammation (high 324 CRP), insufficient sleep, and family history of diabetes or heart attack increased the likelihood of 325 frailty, suggesting a high-stress burden. Our study population had more males but frailty was 326 higher among women. Although women live longer than men, our results agree with other 327 reports, showing that women tend to have poorer health status. Even in developed countries, the environment is more adverse for women, and lifestyle factors may increase women's vulnerability to stochastic subcellular events that increase recovery time (Hubbard and

330 Rockwood, 2015). In our study, this was evident in the mean age of frail females, which was 73.7 331 years, younger than the 75.4 years of frail males. However, the mortality of frail men exceeded 332 that of women. Major causes of death among frail women were chronic kidney diseases, of which 333 all stages are associated with frailty (Wilhelm-Leen et al. 2009), and chronic lower respiratory 
334 diseases. These leading causes of death are longer, slower disease processes that contribute to 335 progressive debilitation, helping to explain why mortality among frail women is lower than in 336 frail males.

\section{Strengths and Limitations}

338 The present study is strengthened by the use of NHANES, which is a well-known and often used national health survey and one of the few population-based surveys that include validated examination measures, biological specimen collection, and limited measures of health status. The analysis in the present study was conducted in a nationally representative sample, allowing our results to be generalized to the entire U.S. adult population. This study also has some limitations, including that it used cross-sectional analysis, which limits inferences regarding causality. Also, the FRAIL scale was used for evaluating frailty among NHANES participants, and it is not known whether other scales may have produced different results. Although frailty is considered a predictor of all-cause mortality (Kulmala et al. 2014; Lin et al.2016), only a small number of participants were included in the deceased/mortality groups categorized by frailty, which limited our findings and precluded making comparisons relative to cause of death as reported in the above-mentioned studies. A major limitation is that the sample is not geographically representative of the United States, even while it is demographically representative; because the two NHANES teams could only visit 16 places each year, achieving a good geographic spread was not possible. NHANES data also do not represent observed changes over time. In-person interview data (by questionnaire) were based on self-reports and subject to recall problems, misunderstanding of questions, and various other factors. NHANES is US data (including representative proportions of different ethnic groups) and needs to be validated in other countries. 
357 Older adults categorized as frail have higher mortality rates than those who are pre-frail or non-

358 frail. Factors associated with frailty differ by gender, with higher frailty prevalence in females

359 and higher mortality in males. Although numbers are small in the present study, the leading

360 causes of death among frail older adults are heart diseases in males and chronic kidney diseases

361 in females. Gender-associated factors for frailty identified in this study may be useful in

362 evaluating frailty and guiding development of public health measures for prevention.

\section{Acknowledgements}

365 The authors wish to thank the US National Center for Health Statistics (NCHS) for creating the

366 National Health and Nutrition Examination Survey and making the NHANES data available to

367 researchers. We also acknowledge that interpreting and reporting these data are the sole

368 responsibility of the authors. 


\section{REFERENCES}

370

371

372

373

374

375

376

377

378

379

380
Ainsworth BE, Haskell WL, Herrmann SD, Meckes N, Bassett DR, Jr., Tudor-Locke C, Greer JL, Vezina J, Whitt-Glover MC, Leon AS. 2011. 2011 Compendium of Physical Activities: a second update of codes and MET values. Medicine and Science in Sports and Exercise. 43:1575-1581. 10.1249/MSS.0b013e31821ece12

Blodgett J, Theou O, Kirkland S, Andreou P, Rockwood K. 2015a. The association between sedentary behaviour, moderate-vigorous physical activity and frailty in NHANES cohorts. Maturitas. 80:187-191. 10.1016/j.maturitas.2014.11.010

Blodgett J, Theou O, Kirkland S, Andreou P, Rockwood K. 2015b. Frailty in NHANES: Comparing the frailty index and phenotype. Archives of Gerontology and Geriatrics. 60:464-470. 10.1016/j.archger.2015.01.016

Centers for Disease Control and Prevention (CDC). National Center for Health Statistics(NCHS). National Health and Nutrition Examination Survey Data 2007-2008 Public Data General Release, U.S. Department of Health and Human Services, Centers for Disease Control and Prevention, https://www.cdc.gov/Nchs/Nhanes/about nhanes.htm.

Chan R, Leung J, Woo J. 2015. Dietary Patterns and Risk of Frailty in Chinese CommunityDwelling Older People in Hong Kong: A Prospective Cohort Study. Nutrients. 7:70707084. $10.3390 /$ nu 7085326

Clegg A, Young J, Iliffe S, Rikkert MO, Rockwood K. 2013. Frailty in elderly people. Lancet. 381:752-762. 10.1016/s0140-6736(12)62167-9

Eeles EM, White SV, O'Mahony SM, Bayer AJ, Hubbard RE. 2012. The impact of frailty and delirium on mortality in older inpatients. Age Ageing. 41:412-416. 10.1093/ageing/afs021

Eichholzer M, Richard A, Walser-Domjan E, Linseisen J, Rohrmann S. 2013. Urinary phytoestrogen levels and frailty in older American women of the National Health and 
Nutrition Examination Survey (NHANES) 1999-2002: a cross-sectional study. Annals of Nutrition and Metabolism. 63:269-276. 10.1159/000356453

395

Fried LP, Tangen CM, Walston J, Newman AB, Hirsch C, Gottdiener J, Seeman T, Tracy R, Kop WJ, Burke G, McBurnie MA. 2001. Frailty in older adults: evidence for a phenotype. Journals of Gerontology Series A-Biological Sciences and Medical Sciences. 56:M146156.

Gloth FM 3rd, Scheve AA, Shah S, Ashton R, McKinney R. 1999. The Frail Elderly Functional Assessment questionnaire: its responsiveness and validity in alternative settings. Archives of Physical Medicine and Rehabilitation. 80:1572-1576.

Hubbard RE. 2015. Sex differences in frailty. Interdisciplinary Topics in Geriatrics and Gerontology. 41:41-53.

Hubbard RE, Rockwood K, eds. Frailty in Aging: Biological, Clinical and Social Implications. 2015. Basel: Karger: pp. 41-43.

Kulmala J, Nykānen I, Hartikainen S. 2014. Frailty as a predictor of all-cause mortality in older men and women. Geriatrics and Gerontology International. 14:899-905.

Lin SY, Lee WJ, Chou MY, Peng LN, Chiou ST, Chen LK.. 2016. Frailty index predicts all-cause mortality for middle-aged and older Taiwanese: Implications for active-aging programs. PLoS One. 11: e0161456.

Ma L, Tang Z, Sun F, Li Y, Chan P. 2017. Prevalence of frailty and associated factors in the community-dwelling population of China. Journal of the Ameriucan Geriatric Society. Nov. 23. doi:10.1111/jgs.15214. [Epub ahead of print.]

Miller AJ, Theou O, McMillan M, Howlett SE, Tennankore KK, Rockwood K. 2017. Dysnatremia in Relation to Frailty and Age in Community-dwelling Adults in the National Health and Nutrition Examination Survey. Journals of Gerontology Series ABiological Sciences and Medical Sciences. 72:376-381. 10.1093/gerona/glw114 
418 Mitnitski AB, Mogilner AJ, Rockwood K. 2001. Accumulation of deficits as a proxy measure of 419 aging. Scientific World Journal. 1:323-336. 10.1100/tsw.2001.58

420

421

422

423

Morley JE, Malmstrom TK, Miller DK. 2012. A simple frailty questionnaire (FRAIL) predicts outcomes in middle aged African Americans. Journal of Nutrition, Health \& Aging. 16:601-608.

National Health and Nutrition Examination Survey (NHANES) Body Mass Index data collection. U.S. Department of Health and Human Services CfDCaPhw.

National Health and Nutrition Examination Survey (NHANES) Laboratory Data Protocol. U.S. Department of Health and Human Services CfDCaP, https://wwwn.cdc.gov/Nchs/Nhanes/2007-2008/DRIIFF_E.htm (Accessed February 12, 2017.)

National Center for Health Statistics (NCHS) Research Ethics Review

https://www.cdc.gov/nchs/nhanes/irba98.htm (Accessed October 12, 2017)

National Institute on Alcohol Abuse and Alcoholism. Helping patients who drink too much: A clinician's guide. NIH Publication No 05-3769. Bethesda M, USA: National Institutes of Health, 2005.

National Sleep Foundation (NSF). http://sleepfoundation.org/how-sleep-works/how-much-sleepdo-we-really-need (Accessed February 12, 2017).

Rockwood K, Mitnitski A, Song X, Steen B, Skoog I. 2006. Long-term risks of death and institutionalization of elderly people in relation to deficit accumulation at age 70. Journal of the American Geriatrics Society. 54:975-979. 10.1111/j.1532-5415.2006.00738.x

Rodriguez-Manas L, Fried LP. 2015. Frailty in the clinical scenario. Lancet. 385:e7-9. $10.1016 / \mathrm{s} 0140-6736(14) 61595-6$ 
441 Romero-Ortuno R, Fouweather T, Jagger C. 2014. Cross-national disparities in sex differences in 442 life expectancy with and without frailty. Age Ageing. 43:222-228.

443 Schleicher RL, Sternberg MR, Lacher DA, Sempos CT, Looker AC, Durazo-Arvizu RA, Yetley

Song X, Mitnitski A, Rockwood K. 2010. Prevalence and 10-year outcomes of frailty in older adults in relation to deficit accumulation. Journal of the American Geriatrics Society. 58:681-687. 10.1111/j.1532-5415.2010.02764.x

Sternberg SA, Wershof Schwartz A, Karunananthan S, Bergman H, Mark Clarfield A. 2011. The identification of frailty: a systematic literature review. Journal of the American Geriatrics Society. 59:2129-2138. 10.1111/j.1532-5415.2011.03597.x

Theou O, Rockwood MR, Mitnitski A, Rockwood K. 2012. Disability and co-morbidity in relation to frailty: how much do they overlap? Archives of Gerontology and Geriatrics. 55:e1-8. 10.1016/j.archger.2012.03.001

U.S. Department of Agriculture and U.S. Department of Health and Human Services. Dietary Guidelines for Americans, Washington, DC: U.S. Government Printing Office, December 2010.

Walston J, Hadley EC, Ferrucci L, Guralnik JM, Newman AB, Studenski SA, Ershler WB, Harris T, Fried LP. 2006. Research agenda for frailty in older adults: toward a better 
466

467

468

469

470

471

472

473

474

475

476

477

478

479

480

481

482

483 understanding of physiology and etiology: summary from the American Geriatrics Society/National Institute on Aging Research Conference on Frailty in Older Adults. Journal of the American Geriatrics Society. 54:991-1001. 10.1111/j.15325415.2006.00745.x

Wang C, Song X, Mitnitski A, Yu P, Gang X, Tang Z, Shi J, Rockwood K. 2013 Gender differences in the relationship between smoking and frailty: results from the Beijing Longitudinal Study of Aging. Journals of Gerontology Series A-Biological Sciences and Medical. 68:338-346.

Waters DL, van Kan GA, Cesari M, Vidal K, Rolland Y, Vellas B. 2012. Gender specific associations between frailty and body composition. Journal of Frailty in Aging. 1:18-23.

Wilhelm-Leen ER, Hall YN, K Tamura M, Chertow GM. 2009. Frailty and chronic kidney disease: the Third National Health and Nutrition Evaluation Survey. American Journal of Medicine. 122:664-671 e662. 10.1016/j.amjmed.2009.01.026

World Health Organization. Metabolic Equivalent of Task (MET) scores: http://www.who.int/chp/steps/GPAQ/en/(Accessed February 12.

Yamada M, Arai H. 2015. Predictive Value of Frailty Scores for Healthy Life Expectancy in Community-Dwelling Older Japanese Adults. Journal of the American Medical Directors Association. 16:1002 e1007-1011. 10.1016/j.jamda.2015.08.001 


\section{Table legends}

485 Table 1. Subjects' demographic and anthropometric characteristics and personal/family medical 486 history

487 Table 2. Subjects' dietary intake, laboratory data and behavioral factors by gender

488 Table 3. Causes of death

489 Table 4 Factors associated with frailty: univariate ordinal regression analysis stratified by 490 gender

\section{Figure legend}

492 Figure 1. Risk factors for geriatric frailty: multivariable regression analysis in males (A) and 493 females (B) 


\section{Figure 1}

Risk factors for geriatric frailty: multivariable regression analysis in males $(A)$ and females (B)

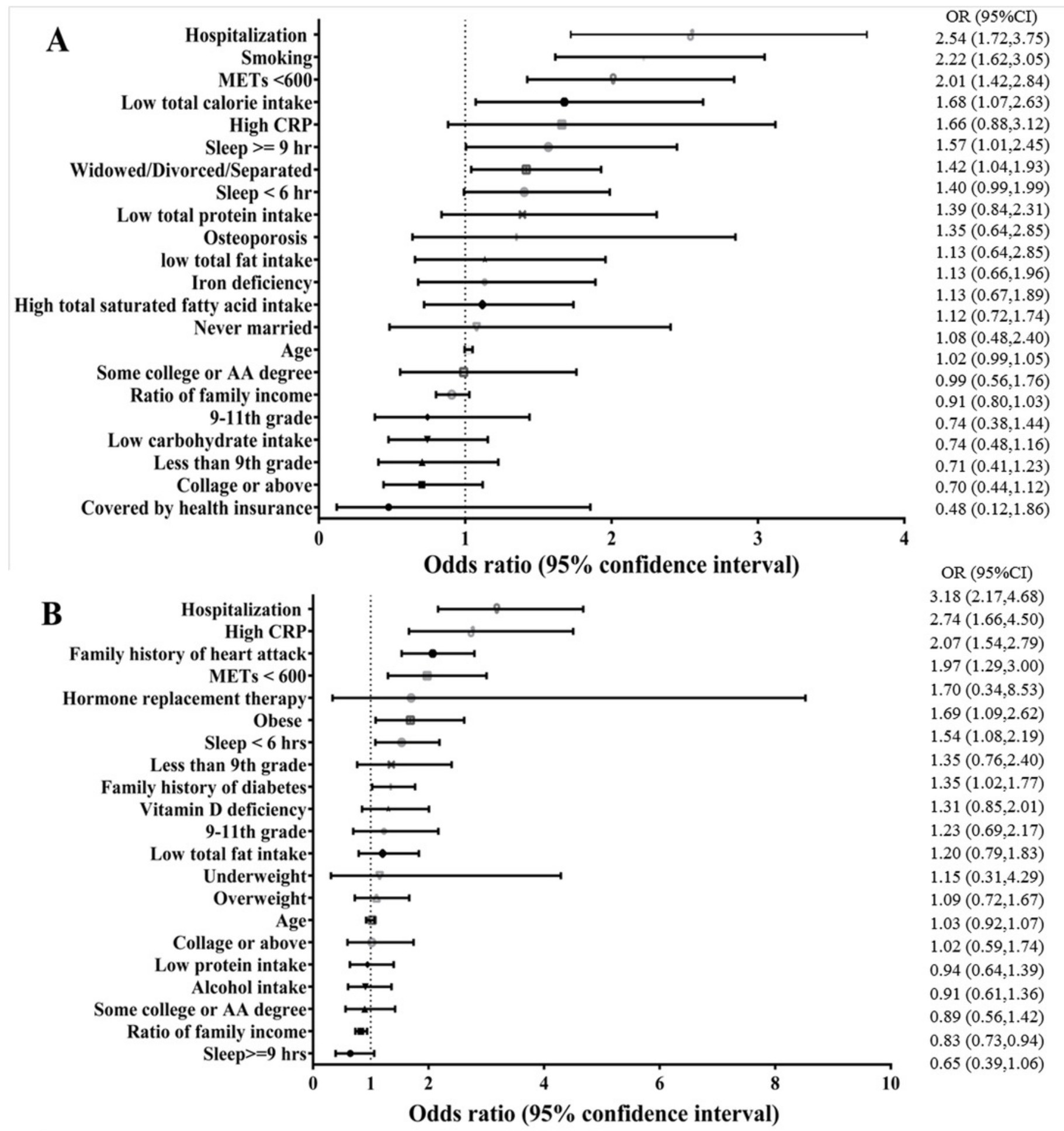




\section{Table $\mathbf{1}$ (on next page)}

Subjects' demographic and anthropometric characteristics and personal/family medical history 
Table 1. Subjects' demographic and anthropometric characteristics and personal/family medical history by gender and frailty cluster

\begin{tabular}{|c|c|c|c|c|c|c|c|c|}
\hline \multirow[b]{2}{*}{ Variables } & \multicolumn{3}{|c|}{ Male $(n=1005)$} & \multicolumn{4}{|c|}{ Female $(\mathrm{n}=948)$} & \multirow[b]{2}{*}{ p-value } \\
\hline & $\begin{array}{l}\text { Non-frail } \\
(\mathrm{n}=567)\end{array}$ & $\begin{array}{l}\text { Pre-frail } \\
(\mathrm{n}=383)\end{array}$ & $\begin{array}{l}\text { Frail } \\
(\mathrm{n}=55)\end{array}$ & p-value & $\begin{array}{l}\text { Non-frail } \\
(\mathrm{n}=485)\end{array}$ & $\begin{array}{l}\text { Pre-frail } \\
(\mathrm{n}=380)\end{array}$ & $\begin{array}{l}\text { Frail } \\
(n=83)\end{array}$ & \\
\hline \multicolumn{9}{|l|}{ Demographic features } \\
\hline Age, years & $71.96 \pm 0.22$ & $72.58 \pm 0.29$ & $75.38 \pm 1.14$ & $0.001 *$ & $72.47 \pm 0.30$ & $73.66 \pm 0.38$ & $73.70 \pm 0.77$ & $0.020^{*}$ \\
\hline Race/ethnicity & & & & 0.479 & & & & 0.140 \\
\hline Mexican American & $45(2.9)$ & $45(4.8)$ & $3(2.5)$ & & $48(2.9)$ & $49(3.9)$ & $4(1.5)$ & \\
\hline Non-Hispanic White & $370(84.1)$ & $242(82.2)$ & $43(86.8)$ & & $328(87.7)$ & $230(83.0)$ & $53(84.5)$ & \\
\hline Non-Hispanic Black & $87(6.3)$ & $62(7.7)$ & $5(3.8)$ & & $63(6.2)$ & $61(8.3)$ & $19(10.9)$ & \\
\hline Other Hispanic & $44(2.7)$ & $24(2.5)$ & $2(2.0)$ & & $42(2.4)$ & $32(2.6)$ & $6(2.4)$ & \\
\hline Other race & 21(3.9) & $10(2.8)$ & $2(4.8)$ & & $4(0.8)$ & $8(2.2)$ & $1(0.7)$ & \\
\hline Marital status & & & & $0.024 *$ & & & & 0.169 \\
\hline Married/Living with partner & $442(82.7)$ & $265(73.9)$ & $42(79.8)$ & & $242(56.2)$ & $162(47.2)$ & $36(52.8)$ & \\
\hline Widowed/Divorced/Separated & $103(14.0)$ & $108(23.6)$ & $11(17.4)$ & & $226(41.5)$ & 205(49.9) & $43(44.3)$ & \\
\hline Never married & $22(3.3)$ & $10(2.4)$ & $2(2.8)$ & & $17(2.2)$ & $13(2.9)$ & $4(2.9)$ & \\
\hline BMI ${ }^{\mathbf{1}, 2}$ & & & & 0.101 & & & & $<0.001$ \\
\hline Underweight & $5(0.8)$ & $6(1.0)$ & $1(3.0)$ & & $6(1.8)$ & $6(1.5)$ & $1(1.5)$ & \\
\hline
\end{tabular}




\begin{tabular}{|c|c|c|c|c|c|c|c|c|}
\hline Overweight & $260(45.0)$ & $150(36.5)$ & $22(43.3)$ & & $183(38.0)$ & $130(34.6)$ & $24(28.8)$ & \\
\hline Obese & $150(28.7)$ & $143(39.6)$ & $21(36.4)$ & & $133(25.0)$ & $141(33.8)$ & $44(55.5)$ & \\
\hline Normal & $150(25.2)$ & $79(21.9)$ & $10(16.3)$ & & $162(35.0)$ & $99(28.7)$ & $13(13.7)$ & \\
\hline Family income/poverty ratio & $3.34 \pm 0.07$ & $2.94 \pm 0.10$ & $2.65 \pm 0.15$ & $<0.001^{*}$ & $3.09 \pm 0.10$ & $2.53 \pm 0.10$ & $2.36 \pm 0.20$ & $<0.001$ \\
\hline Education $^{1}$ & & & & $0.019^{*}$ & & & & 0.081 \\
\hline Less than 9th grade & $82(8.3)$ & $72(10.5)$ & $10(13.6)$ & & $63(5.4)$ & $75(11.9)$ & $16(11.6)$ & \\
\hline 9-11th grade & $74(10.5)$ & $57(10.7)$ & $10(17.0)$ & & $67(13.0)$ & $77(19.2)$ & $20(19.0)$ & \\
\hline High school grad & $133(22.0)$ & $91(28.0)$ & $12(27.0)$ & & $134(29.4)$ & $102(29.7)$ & $23(34.1)$ & \\
\hline Some college or AA degree & $110(22.0)$ & $89(26.7)$ & $14(26.6)$ & & $124(28.2)$ & $82(23.5)$ & $15(20.9)$ & \\
\hline College graduate or above & $168(37.3)$ & $74(24.0)$ & $9(15.8)$ & & $95(23.5)$ & $44(15.6)$ & $9(14.3)$ & \\
\hline Health insurance status & & & & 0.093 & & & & 0.568 \\
\hline Covered by health insurance & $557(99.4)$ & $374(98.4)$ & $52(96.8)$ & & $471(98.4)$ & $364(98.2)$ & $82(99.5)$ & \\
\hline Not covered by health insurance & $9(0.6)$ & $8(1.6)$ & $3(3.2)$ & & $14(1.6)$ & $16(1.8)$ & $1(0.5)$ & \\
\hline \multicolumn{9}{|l|}{ amily/personal medical history } \\
\hline Hormone replacement therapy & $\mathrm{N} / \mathrm{A}$ & $\mathrm{N} / \mathrm{A}$ & $\mathrm{N} / \mathrm{A}$ & & $232(54.4)$ & $149(44.1)$ & $25(41.3)$ & $0.024^{*}$ \\
\hline Close relative had asthma & $64(9.7)$ & $51(10.7)$ & $6(14.3)$ & 0.076 & $59(15.0)$ & $62(15.6)$ & $14(21.0)$ & 0.514 \\
\hline Close relative had diabetes & $177(30.5)$ & $132(35.7)$ & $24(35.8)$ & 0.080 & $55(12.8)$ & $63(18.6)$ & $15(20.8)$ & 0.058 \\
\hline Close relative had heart attack & $59(12.0)$ & $42(12.6)$ & $8(15.6)$ & 0.180 & $165(31.1)$ & $152(39.3)$ & $33(37.7)$ & $0.019 *$ \\
\hline
\end{tabular}




\begin{tabular}{|c|c|c|c|c|c|c|c|c|}
\hline Close relative had osteoporosis & $31(6.2)$ & $22(7.9)$ & $3(5.5)$ & 0.272 & $64(16.0)$ & $41(11.3)$ & $13(20.5)$ & 0.114 \\
\hline Hospitalization $^{1}$ & & & & $<0.001 *$ & & & & $<0.001$ \\
\hline$>3$ times & $0(0)$ & $1(0.3)$ & $2(5.5)$ & & $0(0)$ & $2(0.7)$ & $2(2.6)$ & \\
\hline$<3$ times & $71(14.0)$ & $80(22.8)$ & $23(42.9)$ & & $48(8.7)$ & $74(19.4)$ & $26(30.9)$ & \\
\hline No hospitalization & $496(86.0)$ & $302(76.8)$ & $30(51.6)$ & & $437(91.3)$ & $304(79.9)$ & $54(65.8)$ & \\
\hline Mental health consultation & $6(1.1)$ & $10(2.7)$ & $1(2.4)$ & 0.243 & $6(1.6)$ & $7(2.0)$ & $4(4.0)$ & 0.468 \\
\hline Osteoporosis $^{1}$ & $13(2.7)$ & $21(6.4)$ & $1(2.8)$ & $0.007^{*}$ & $130(29.1)$ & $84(22.6)$ & $23(29.8)$ & 0.243 \\
\hline Fracture & $62(12.0)$ & $50(14.9)$ & $13(23.3)$ & 0.107 & $66(13.1)$ & $54(15.5)$ & $19(25.1)$ & 0.114 \\
\hline Steroid usage $^{1}$ & $23(5.3)$ & $18(5.1)$ & $10(19.2)$ & $0.001 *$ & $33(8.1)$ & $20(4.9)$ & $5(6.7)$ & 0.388 \\
\hline
\end{tabular}




\section{Table 2(on next page)}

Subjects' dietary intake, laboratory data and behavioral factors by gender 
Table 2. Subjects' dietary intake, laboratory data and behavioral factors by gender and frailty cluster

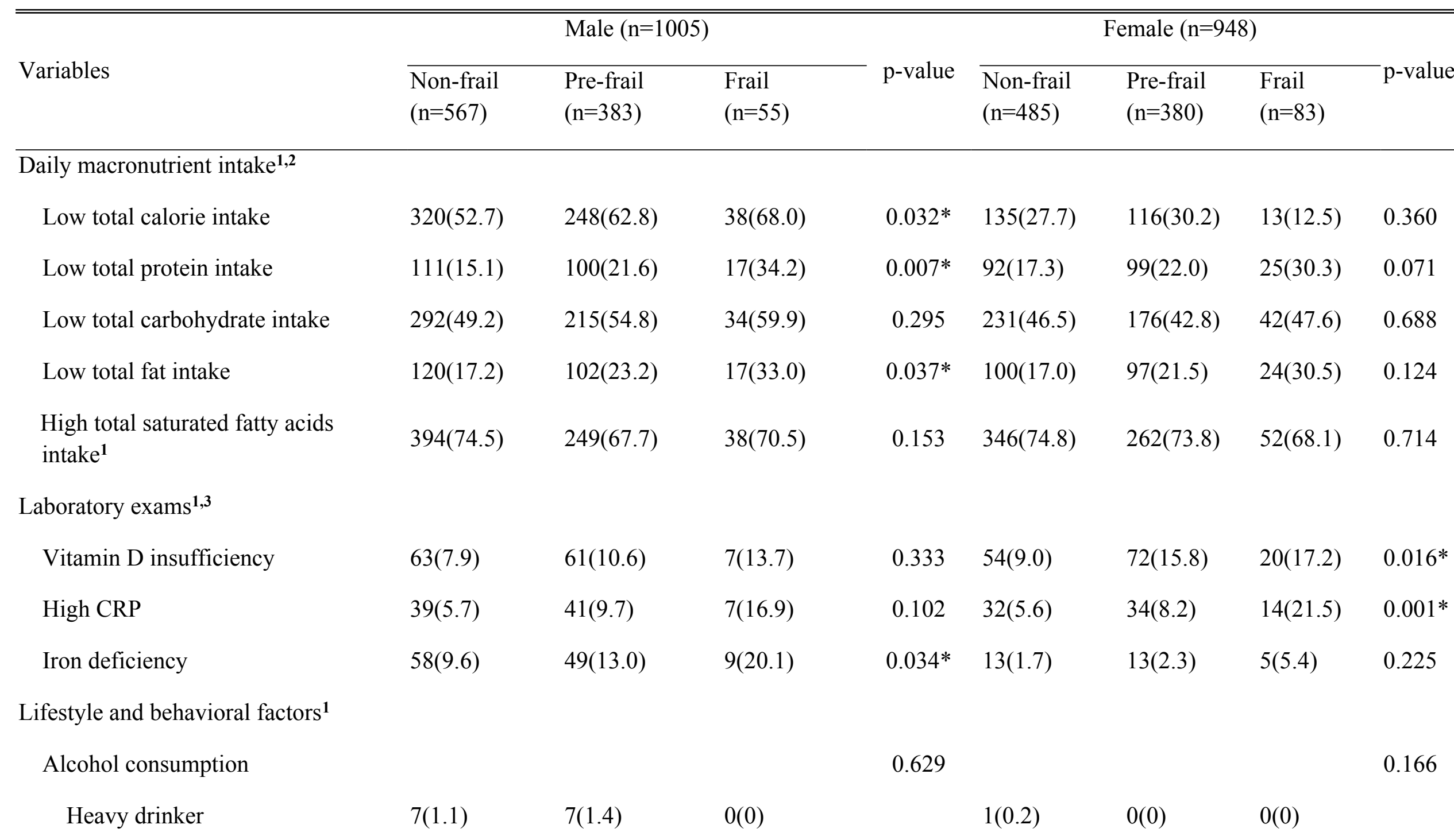


Moderate drinker

Occasional

Milk consumption

$$
\text { Regular }
$$

Never regular

Sometimes regular

Vegetarian

Food allergies

Water devices use

Physical activity (MET minutes)

$$
\begin{aligned}
& <600 \\
& >=600
\end{aligned}
$$

Sleeping hours

$$
\begin{aligned}
& <6 \mathrm{hr} . \\
& >=9 \mathrm{hr} . \\
& 7-8 \mathrm{hr} .
\end{aligned}
$$

Smoking

$$
\begin{array}{lll}
446(79.0) & 301(81.4) & 44(81.7) \\
111(19.5) & 74(17.0) & 11(18.3)
\end{array}
$$

0.172

$\begin{array}{lll}277(50.8) & 178(47.5) & 30(51.4) \\ 123(18.6) & 77(20.9) & 4(5.3) \\ 167(30.6) & 128(31.7) & 21(43.3)\end{array}$

12(1.2) $7(1.3)$

$38(7.9)$

21(5.6)

$188(41.2)$

106(33.5)

$$
0(0)
$$

$2(2.5)$

19(38.2)

$$
\text { 213(31.0) }
$$$$
\text { 183(44.0) }
$$$$
\text { 47(87.4) }
$$$$
354(69.0)
$$

$200(56.0)$

$8(12.6)$

$0.049 *$

$\begin{array}{lll}170(27.8) & 144(34.2) & 26(46.1) \\ 50(8.4) & 51(13.1) & 6(11.7) \\ 345(63.5) & 188(52.7) & 23(42.2) \\ 326(54.7) & 276(72.1) & 47(86.8)\end{array}$

$\begin{array}{lll}257(57.7) & 162(46.6) & 37(45.6) \\ 227(42.1) & 216(53.0) & 46(54.4)\end{array}$

0.247

$215(44.4) \quad 159(44.4) \quad 25(29.8)$

122(25.3) $\quad 88(23.6) \quad 26(25.4)$

$148(30.2) \quad 133(32.0) \quad 32(44.8)$

$\begin{array}{llll}0.724 & 7(1.0) & 10(2.7) & 4(5.2)\end{array}$

0.164

$\begin{array}{lllll}0.437 & 62(12.9) & 50(12.0) & 10(16.1) & 0.732\end{array}$

$\begin{array}{lllll}0.572 & 162(38.8) & 109(31.9) & 25(38.8) & 0.632\end{array}$

$<0.001 *$

$<0.001^{\prime}$

$\begin{array}{lll}238(44.0) & 255(63.5) & 65(73.8) \\ 247(56.0) & 125(36.5) & 18(26.2)\end{array}$

135(25.7) $\quad 150(34.7) \quad 34(42.5)$

$39(8.8) \quad 42(11.4) \quad 1(0.5)$

$311(65.5) \quad 188(54.0) \quad 48(57.0)$

$<0.001 * \quad 164(35.2) \quad 114(33.2) \quad 32(32.9)$ 
2. According to 2015-2020 Dietary Guidelines for Americans, the recommended daily allowances (RDA) for nutrients are as follows: calorie intake between 2000 2800 Kcal for males and 1600 2200 Kcal for females; protein intake between 50 245 gm for males and 40 192.5 gm for females; carbohydrate intake between $225 \sim 455$ gm for males and 180 357.5 gm for females; total fat intake between 44.4 109 gm for males and 35.5 85.5 gm for females; saturated fatty acids intake $\leq 15.6$ gm for males and $\leq 12.3$ gm for females.

3. Normal serum levels are defined as: serum 25-hydroxyvitamin D (25[OH]D) $\geq 40 \mathrm{nmol} / \mathrm{L}$; CRP between 0-1 mg/dL; iron level between $55-160 \mu \mathrm{g} / \mathrm{dL}$ for males and $40-155 \mu \mathrm{g} / \mathrm{dL}$ for females.

10 Asterisk indicates statistical difference between groups, $\mathrm{p}<0.05$. 
Table 3 (on next page)

Causes of death 
Table 3. Causes of death by gender and frailty cluster

\begin{tabular}{|c|c|c|c|c|c|c|}
\hline \multirow{2}{*}{ Variables } & \multicolumn{3}{|c|}{ Male $(n=1005)$} & \multicolumn{3}{|c|}{ Female $(n=948)$} \\
\hline & $\begin{array}{c}\text { Non-frail } \\
(\mathrm{n}=567)\end{array}$ & $\begin{array}{l}\text { Pre-frail } \\
(n=383)\end{array}$ & $\begin{array}{c}\text { Frail } \\
(n=55)\end{array}$ & $\begin{array}{l}\text { Non-frail } \\
(\mathrm{n}=485)\end{array}$ & $\begin{array}{l}\text { Pre-frail } \\
(n=380)\end{array}$ & $\begin{array}{l}\text { Frail } \\
(n=83)\end{array}$ \\
\hline \multicolumn{7}{|l|}{ Survival status } \\
\hline \multirow{2}{*}{ Alive } & $531(94.6$ & $346(91.3$ & $44(77.5)$ & $470(97.5$ & $356(93.6$ & $76(91.5$ \\
\hline & ) & ) & & ) & ) & ) \\
\hline Deceased & $36(5.4)$ & $37(8.7)$ & $11(22.5)$ & $15(2.5)$ & $24(6.4)$ & $7(8.5)$ \\
\hline Heart diseases & $7(27.2)$ & $9(19.5)$ & $4(41.0)$ & $3(8.1)$ & $7(24.0)$ & $0(0)$ \\
\hline Malignant neoplasms & $17(43.2)$ & $12(39.9)$ & $2(16.3)$ & $4(24.0)$ & $5(23.0)$ & $1(11.3)$ \\
\hline Chronic lower respiratory diseases & $1(2.2)$ & $0(0)$ & $2(23.0)$ & $0(0)$ & $3(17.0)$ & $1(17.6)$ \\
\hline Accidents (unintentional injuries) & $0(0)$ & $1(1.2)$ & $0(0)$ & $0(0)$ & $0(0)$ & $0(0)$ \\
\hline Cerebrovascular diseases & $4(7.6)$ & $0(0)$ & $0(0)$ & $1(9.5)$ & $0(0)$ & $1(13.3)$ \\
\hline Alzheimer disease & $1(1.2)$ & $0(0)$ & $0(0)$ & $0(0)$ & $1(5.0)$ & $0(0)$ \\
\hline Diabetes mellitus & $0(0)$ & $1(2.8)$ & $0(0)$ & $0(0)$ & $2(6.4)$ & $0(0)$ \\
\hline Influenza and pneumonia & $0(0)$ & $2(7.5)$ & $0(0)$ & $1(1.8)$ & $0(0)$ & $1(10.7)$ \\
\hline Nephritis, nephrotic syndrome and nephrosis & $1(3.2)$ & $0(0)$ & $0(0)$ & $0(0)$ & $0(0)$ & $2(32.3)$ \\
\hline All other causes & $5(15.4)$ & $12(29.1)$ & $3(19.7)$ & $6(56.6)$ & $6(24.7)$ & $1(14.8)$ \\
\hline
\end{tabular}




\section{Table 4(on next page)}

Factors associated with frailty: univariate ordinal regression analysis stratified by gender 
1 Table 4 Factors associated with frailty: univariate ordinal regression analysis stratified by gender

\begin{tabular}{|c|c|c|c|c|}
\hline Univariate & \multicolumn{2}{|l|}{ Male } & \multicolumn{2}{|l|}{ Female } \\
\hline Age & $1.042(1.018,1.066)$ & $0.001 *$ & $1.041(1.013,1.07)$ & $0.006^{*}$ \\
\hline Mexican American & $1.476(0.981,2.22)$ & 0.062 & $1.118(0.76,1.646)$ & 0.559 \\
\hline Non-Hispanic Black & $1.114(0.775,1.601)$ & 0.556 & $1.519(0.957,2.412)$ & 0.088 \\
\hline Other Hispanic & $0.904(0.505,1.62)$ & 0.736 & $1.094(0.642,1.863)$ & 0.742 \\
\hline Widowed/Divorced/Separated & $1.714(1.274,2.305)$ & $0.001 *$ & $1.31(0.944,1.816)$ & 0.106 \\
\hline Never married & $0.830(0.368,1.875)$ & 0.660 & $1.462(0.768,2.783)$ & 0.248 \\
\hline \multicolumn{5}{|l|}{ BMI (Ref= Normal) } \\
\hline Underweight & $2.069(0.243,17.61)$ & 0.539 & $1.207(0.332,4.386)$ & 0.777 \\
\hline Overweight & $1.008(0.68,1.494)$ & 0.968 & $1.224(0.89,1.683)$ & 0.216 \\
\hline
\end{tabular}


Obese

Family poverty/income ratio

Education $($ Ref $=$ High school grad $)$

Less than 9th grade

9-11th grade

Some college or AA degree

College graduate or above

Health insurance coverage

Daily macronutrient intake $(\mathrm{Ref}=$ higher /recommended)

Low total calorie intake

Low total protein intake

Low total carbohydrate intake

Low total fat intake

High saturated fatty acids intake

Laboratory exams $(\operatorname{Ref}=$ Normal $)$

Vitamin D insufficiency

High CRP

$\begin{array}{ll}1.612(0.994,2.614) & 0.059 \\ 0.819(0.739,0.907) & 0.001 *\end{array}$

$2.263(1.635,3.132)$

$<0.001^{*}$

$0.758(0.668,0.86)$

$<0.001^{*}$

$\begin{array}{llll}1.065(0.649,1.746) & 0.805 & 1.851(1.157,2.963) & 0.010^{*} \\ 0.905(0.55,1.489) & 0.701 & 1.344(0.82,2.202) & 0.241 \\ 0.959(0.562,1.638) & 0.880 & 0.772(0.521,1.146) & 0.199 \\ 0.488(0.338,0.706) & 0.001^{*} & 0.622(0.373,1.037) & 0.069 \\ 0.336(0.128,0.882) & 0.036^{*} & 1.091(0.456,2.609) & 0.838\end{array}$

$0.336(0.128,0.882)$

$.036^{*}$

(0.456, 2.609)

0.838

$\begin{array}{llll}1.559(1.176,2.067) & 0.004^{*} & 1.078(0.804,1.446) & 0.618 \\ 1.756(1.326,2.325) & <0.001^{*} & 1.523(1.118,2.075) & 0.013^{*} \\ 1.285(0.973,1.698) & 0.097 & 0.919(0.663,1.274) & 0.617 \\ 1.591(1.132,2.235) & 0.014^{*} & 1.541(1.101,2.158) & 0.018^{*} \\ 0.729(0.565,0.94) & 0.019^{*} & 0.867(0.598,1.256) & 0.458 \\ & & & \\ 1.489(0.865,2.566) & 0.162 & 1.889(1.266,2.818) & 0.003^{*} \\ 2.053(1.081,3.901) & 0.038^{*} & 2.492(1.5,4.141) & 0.002^{*}\end{array}$


Iron deficiency

Lifestyle and behavioral factors

Alcohol consumption

$(\mathrm{Ref}=$ Occasional $)$

Heavy drinker

Moderate drinker

Milk consumption

(Ref= Regular)

Never regular

Sometimes regular

Vegetarian $(\operatorname{Ref}=\mathrm{No})$

Food allergies $(\mathrm{Ref}=\mathrm{Yes})$

Water devices use $(\mathrm{Ref}=\mathrm{No})$

Physical inactivity (METs $<600)$

(Ref: METs $>=600$ )

Sleeping hours (Ref=7-8 hr.)

$<6 \mathrm{hr}$.

$>=9 \mathrm{hr}$.
$1.614(1.081,2.411)$

$0.019^{*}$

$2.002(0.81,4.944)$

0.163

$\begin{array}{llll}1.181(0.347,4.021) & 0.781 & \mathrm{~N} / \mathrm{A} & \\ 1.164(0.909,1.491) & 0.239 & 0.648(0.437,0.962) & 0.040^{*}\end{array}$

$\begin{array}{llll}1.023(0.685,1.528) & 0.910 & 1.053(0.784,1.414) & 0.732 \\ 1.164(0.82,1.652) & 0.406 & 1.308(0.83,2.06) & 0.255 \\ 0.892(0.318,2.501) & 0.829 & 3.174(0.696,14.477) & 0.136 \\ 1.586(0.762,3.301) & 0.221 & 0.963(0.518,1.792) & 0.908 \\ 0.754(0.511,1.110) & 0.152 & 0.821(0.573,1.177) & 0.283 \\ 2.398(1.749,3.288) & <0.001^{*} & 2.416(1.573,3.709) & <0.001^{*} \\ & & & \\ 1.625(1.205,2.19) & 0.003^{*} & 1.677(1.22,2.307) & 0.003^{*} \\ 1.854(1.283,2.679) & 0.002^{*} & 1.094(0.699,1.712) & 0.679\end{array}$


Smoker $($ Ref $=$ Non-smoker $)$

Hormone replacement therapy

$(\operatorname{Ref}=$ No HRT $)$

Close relative had asthma $(\operatorname{Ref}=\mathrm{No})$

Close relative had diabetes $(\mathrm{Ref}=$ No)

Close relative had heart attack

$(\mathrm{Ref}=\mathrm{No})$

Close relative had osteoporosis

$(\mathrm{Ref}=\mathrm{No})$

Hospitalization $(\mathrm{Ref}=$ No hospital utilization $)$

Mental health consultation

(Ref $=$ No consultation)

Osteoporosis $(\mathrm{Ref}=\mathrm{No})$

Fracture $(\operatorname{Ref}=$ No $)$

Steroid usage $(\operatorname{Ref}=$ No $)$
$2.394(1.775,3.23)$

$<0.001 *$

$0.914(0.721,1.159)$

0.464

N/A

$1.226(0.705,2.133)$

0.479

$0.663(0.506,0.87)$

$0.006^{*}$

$1.289(0.975,1.705)$

0.084

$1.191(0.782,1.814)$

0.427

$1.141(0.657,1.981)$

0.646

$1.412(1.079,1.846)$

$0.017^{*}$

$1.196(0.609,2.349)$

0.600

$0.847(0.621,1.155)$

0.324

$2.391(1.652,3.46)$

$<0.001 *$

$3.118(2.236,4.349)$

$<0.001^{*}$

$2.274(0.837,6.177)$

0.104

$1.647(0.654,4.153)$

0.322

$2.005(1.032,3.896)$

$0.038^{*}$

$0.818(0.548,1.222)$

0.343

$1.441(0.949,2.188)$

0.102

$1.496(0.906,2.47)$

0.134

$1.571(0.79,3.122)$

0.247

$0.653(0.352,1.212)$

0.197

2 Abbreviations: CI: confidence interval; MET: metabolic equivalent task; N/A: not available; OR: odds ratio; Ref: reference

3 1. Only one heavy drinker was found among females, so subject was re-classified into moderate drinker group for analysis.

4 Asterisk indicates statistical difference between groups, $\mathrm{p}<0.05$. 